Revista de Matemática: Teoría y Aplicaciones 2004 11(1) : 1-16

CIMPA - UCR - CCSS ISSN: 1409-2433

\title{
HÉRCULES CONTRA LA HIDRA Y LA MUERTE DEL INTERNET
}

\author{
EduARdo Piza Volio*
}

Recibido/Received: 22 Oct 2003

\begin{abstract}
Resumen
Hércules mató a la Hidra de Lerna en una encarnizada batalla, segunda de las labores que le fueron encomendadas en penitencia por sus horrendos crímenes. La Hidra era aquel espantoso y agresivo monstruo mitológico de múltiples cabezas y sangre envenenada, que multiplicaba sus cabezas cada vez que le cortaban una de ellas.

En este artículo se estudian algunos modelos matemáticos acerca de esta interesante batalla épica. También se estudian las conexiones que tiene este problema con las sucesiones ultra-crecientes y recursivas de Goodstein.

Como una aplicación interesante se analiza la próxima e inevitable muerte de otro de los grandes monstruos de nuestra era moderna: la red Internet.
\end{abstract}

Palabras clave: Hércules, la Hidra, sucesiones de Goodstein, Internet, números ordinales, Aritmética de Peano.

\begin{abstract}
Hercules killed the Hydra of Lerna in a bloody battle - the second of the labor tasks imposed upon him in atonement for his hideous crimes. The Hydra was a horrible, aggressive mythological monster with many heads and poisonous blood, whose heads multiplied each time one of them was severed.

This article explores some mathematical methods about this interesting epic battle. A generalization of the original Kirby \& Paris model is proposed. We also study the connection of this model with Goodstein ultra-growing and recursive sequences.

As an interesting application, we next analyze the inevitable death of another huge monster of our modern era: the Internet.
\end{abstract}

Keywords: Hercules, the Hydra, Goodstein sequences, Internet, ordinals numbers, Peano's Arithmetic.

Mathematics Subject Classification: 03E10, 03C62.

${ }^{*}$ Centro de Investigación en Matemática Pura y Aplicada (CIMPA), Universidad de Costa Rica, 2060 San José, Costa Rica. Email: epiza@cariari.ucr.ac.cr. 
E. PIZA

\section{Hércules y la Hidra: un poco de mitología griega}

Según la mitología griega, el semidiós Hércules ${ }^{1}$, hijo extramarital del dios Júpiter ${ }^{2}$ y de la mortal Alcmena ${ }^{3}$, mató a su propia esposa e hijos cuando se encontraba dentro de un estado de "locura temporal" inducida por la diosa $\mathrm{Juno}^{4}$, quien siempre estuvo determinada a hacerle daño a Hércules.

Cuando se despertó de su locura temporal, Hércules se horrorizó y arrepintiéndose de lo que había hecho le rezó al dios Apolo ${ }^{5}$ pidiéndole perdón y guía espiritual. El dios de los oráculos lo perdonó y envió al servicio del rey Euristeo ${ }^{6}$ durante doce años, a cumplir penitencia y trabajos forzados en compensación por sus asesinatos.

Como parte del castigo que se le impuso por estos horrendos crímenes, le encomendaron doce labores tan difíciles de cumplir que parecían imposibles. Afortunadamente Hércules contó con la eventual ayuda de Hermes ${ }^{7}$ y Atenea $^{8}$ en los momentos cuando más los necesitaba. Al final de estas doce labores Hércules llegó a ser, sin ninguna duda, el más grande de los héroes griegos.

La primera de las labores encomendadas a Hércules fue matar al temible León de Nemea. La segunda fue quizás la más peligrosa de todas las labores: matar a la espantosa Hidra, quien era un peligroso monstruo de sangre envenenada y muchas cabezas que vivía en la inmediaciones de Lerna y tenía atemorizada a la población. Hércules se enfrentó a la Hidra en una formidable batalla, cortándole sus cabezas con una espada. Sin embargo, cada vez que Hércules cortaba una cabeza, del cuerpo de la bestia le brotaban muchas más en sustitución. De acuerdo con los registros oficiales, Hércules ganó. Lo que no revelaron los antiguos griegos es que, no importa en qué orden y cuál estrategia empleara Hércules para cortar las cabezas, siempre terminaría aniquilando a la Hidra, aunque como veremos, necesitaría casi toda la historia del tiempo para hacerlo.

\section{Un modelo matemático de las Hidras y de la batalla}

Aunque menos encantador y botánicamente incorrecto, representaremos matemáticamente a la Hidra como un árbol y a Hércules simplemente como una flecha apuntando a una de las cabezas del árbol.

\footnotetext{
${ }^{1}$ Hércules (nombre latino) o Heracles (nombre griego), el más popular de los héroes mitológicos griegos.

${ }^{2}$ Júpiter (nombre latino) o Zeus (nombre griego), padre de los dioses. Venció a los titanes y derribó a su padre Saturno. Dios del cielo, de la luz diurna, del tiempo y de los rayos.

${ }^{3}$ La bella esposa de Anfitrión.

${ }^{4}$ Juno (nombre latino) o Hera (nombre griego) es la celosa esposa de Júpiter, hija de Saturno, diosa del matrimonio.

${ }^{5}$ Apolo, dios de los oráculos, de la medicina de la poesía, de las artes, de los rebaños, del Día y del Sol. Hijo de Júpiter y Letina.

${ }^{6}$ Euristeo, rey de Tirinto y Micenas, era medio hermano de Hércules.

${ }^{7}$ Hermes (nombre griego) o Mercurio (nombre latino), hijo de Júpiter, dios de la elocuencia, mensajero de los dioses, dios de los ladrones. Medio hermano de Hércules.

${ }^{8}$ Atenea (nombre griego) o Minerva (nombre latino), hija de Júpiter, diosa griega de la sabiduría, de las artes, de las ciencias y de la industria.
} 
Definición 1 (Hidra, raíz, cabezas) Una Hidra $H$ es un árbol, esto es, un grafo finito acíclico y conectado, con un nodo fijo llamado la raíz y denotado por raíz $(H)$. Cualquier nodo terminal distinto de la raíz se llama cabeza.

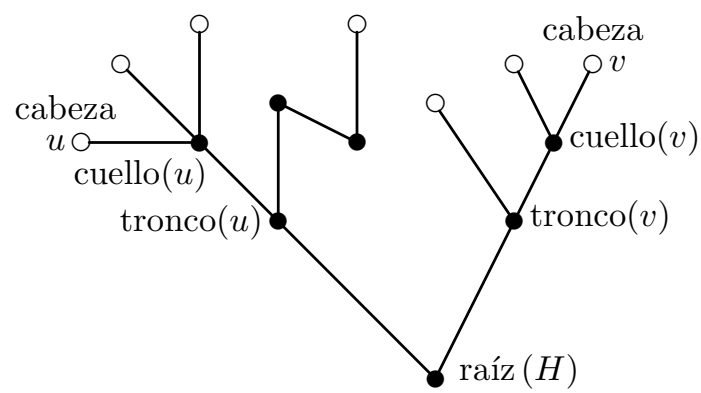

Hidra con 7 cabezas.

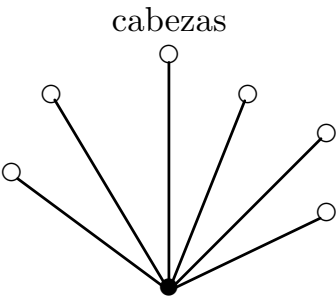

Hidra estrella

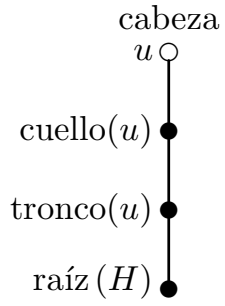

Hidra lineal

Figura 1: Modelo matemático y diferentes tipos de Hidras. Las Hidras estrellas no tienen troncos, tan solo cabezas y la raíz, que coincide con el cuello de las cabezas. Las Hidras lineales solamente tienen una cabeza.

El proceso reproductivo de las cabezas de la Hidra - luego del corte de una de ellaspuede definirse de muy diversas maneras, algunas de las cuales son variaciones más o menos complejas del modelo clásico que explicaremos a continuación, el cual fue introducido por vez primera por Kirby y Paris [3] en 1982.

Definición 2 (Cuello y tronco de una cabeza) Sea $v$ una cabeza de una Hidra H. El nodo anterior a $v$ se llama cuello y se denota por cuello $(v)$. Si cuello $(v)$ tiene a su vez un nodo que lo antecede en la ruta hacia la raíz $(H)$, entonces a este nodo lo llamamos tronco de $v$ y lo denotamos por tronco $(v)$. En caso que cuello $(v)=$ raíz $(H)$, entonces decimos que $v$ no tiene tronco.

La reproducción de las cabezas de la Hidra durante el transcurso de una batalla es la parte más fascinante de este monstruo. Asociada con una cabeza tendremos el cuerpo, que es la sección de la anatomía de la Hidra que se reproduce varias veces cuando la cabeza es cortada. Algunas cabezas no tienen cuerpo asociado.

Definición 3 (Cuerpo de una cabeza) Sea $H$ una Hidra y sea $v$ una cabeza de $H$. Si $v$ tiene tronco, definimos el cuerpo de $v$ (denotado por cuerpo $(v)$ ) como el subárbol que contiene los nodos cuello $(v)$ y todos sus sucesores, eliminando $v$ y luego agregándole el nodo tronco $(v)$ como raíz. En caso que $v$ no tenga tronco, decimos que $v$ no posee cuerpo.

El modelo clásico de reproducción de cabezas de Kirby y Paris viene descrito en la siguiente definición. Una ejemplo de una etapa de la batalla se ilustra en la Figura 3.

Definición 4 (Batalla y reproducción de cabezas) Sea $H$ una Hidra. Una batalla entre Hércules y la Hidra $H$ es una sucesión de Hidras $H_{0}=H, H_{1}, H_{2}, \ldots$ donde la 

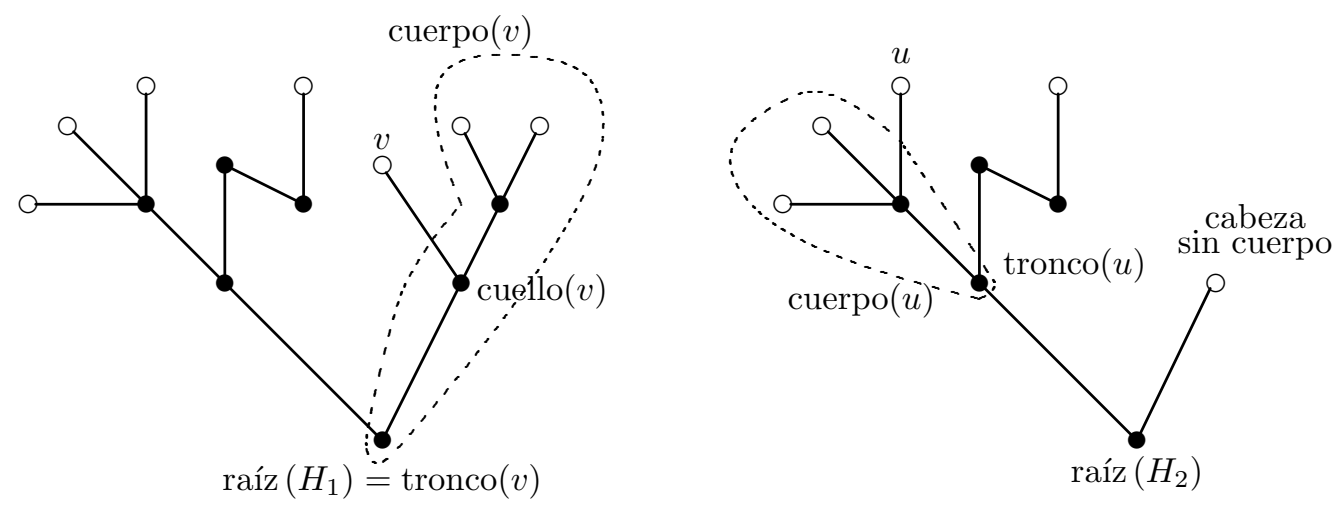

Figura 2: Cuerpos de las cabezas $v$ y $u$ de dos Hidras distintas $H_{1}$ y $H_{2}$.

Hidra $H_{n}$ se obtiene de la anterior, $H_{n-1}$, mediante el siguiente esquema de reproducción: Hércules corta una cabeza cualquiera $v \in H_{n-1}$; en respuesta la Hidra añade $n$ réplicas del cuerpo de $v$, a partir de tronco(v), si v tiene cuerpo. En caso que la cabeza cortada no tenga cuerpo, entonces la Hidra no se reproduce en esa etapa. Hércules gana la batalla si, después de número finito de etapas $k$ (el largo de la batalla), la Hidra $H_{k}$ es justamente la raíz.

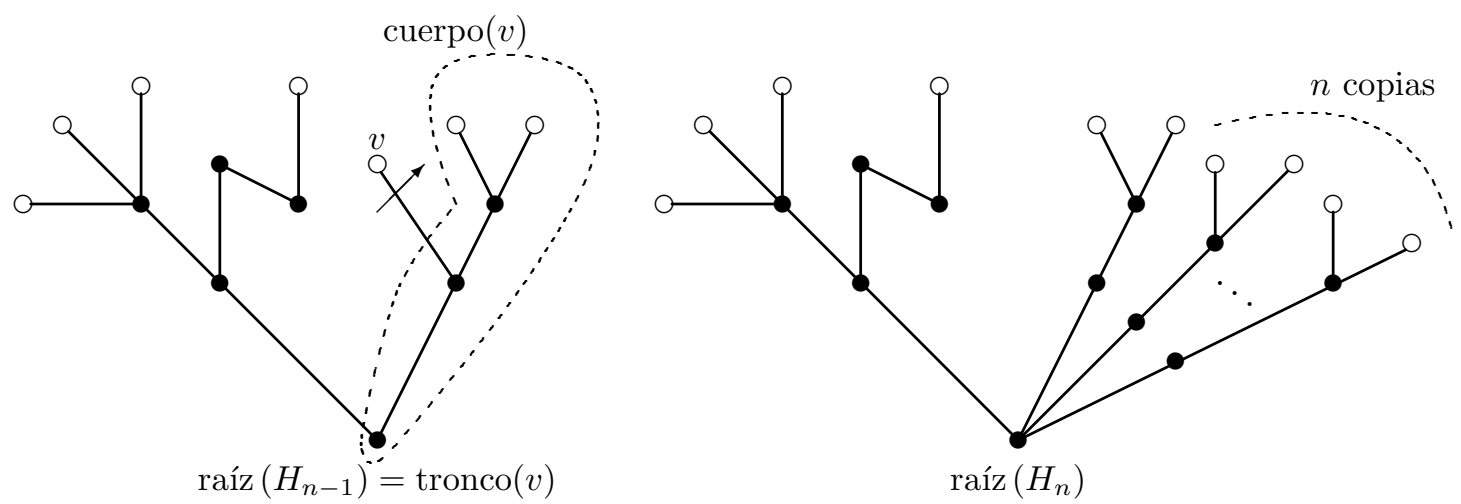

Figura 3: Simulacro de la etapa $n$ de una batalla. A la izquierda Hércules se dispone a cortar la cabeza $v$ de la Hidra $H_{n-1}$. A la derecha se muestra la nueva anatomía de la Hidra $H_{n}$ después de reproducir $n$ copias del cuerpo de la cabeza $v$.

De este modo, las secuencias de una batalla típica entre Hércules y la Hidra podrían desarrollarse, por ejemplo, como se ilustra en la Figura 4. En teoría Hércules tiene la posibilidad de ir escogiendo cuál de las cabezas de la Hidra corta. En cada etapa, la escogencia de la decapitación de una cabeza específica con respecto a otra cualquiera establece las diferentes estrategias que Hércules tiene a su disposición.

Como se indicó en la anterior definición, no siempre la Hidra reproduce nuevas cabezas cuando le cortan una de ellas. La reproducción se lleva a cabo siempre y cuando la cabeza cortada tenga un cuerpo asociado. Pero si la cabeza cortada carece de cuerpo (como por ejemplo en las Hidras estrellas), entonces no se reproducen nuevas cabezas. Esto se ilustra en la Figura 5. 


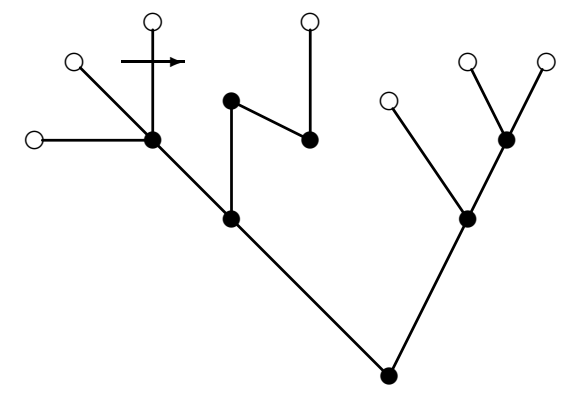

Etapa 1.

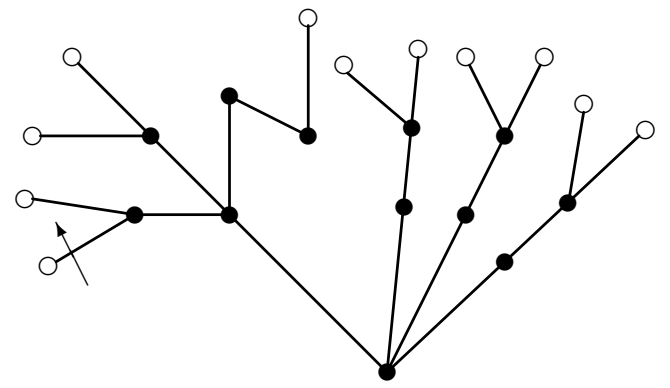

Después de la etapa 2.

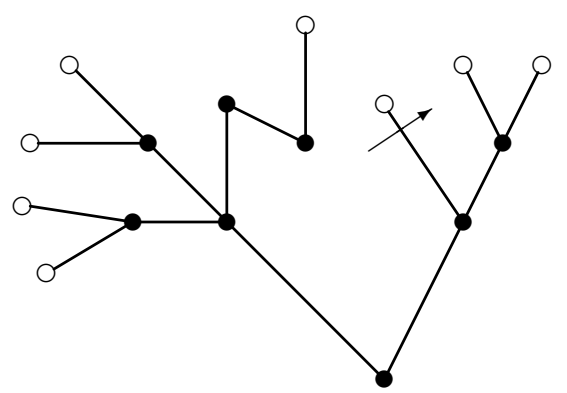

Después de la etapa 1.

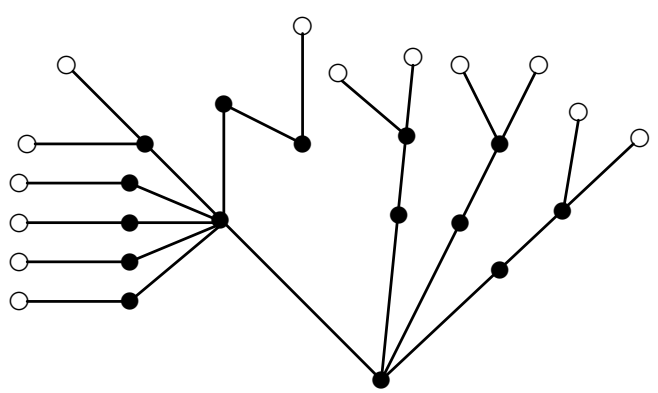

Después de la etapa 3.

Figura 4: Primeras secuencias de una batalla típica entre Hércules y una Hidra.

La muerte de la Hidra se obtendrá luego que Hércules llegue a cortar la última de sus cabezas. Esta última cabeza no se reproducirá, pues no tiene cuerpo asociado. Diremos que una estrategia de Hércules es ganadora si la batalla correspondiente termina con la muerte de la Hidra. En apariencia, esta situación difícilmente se alcanzará, en virtud del crecimiento desmesurado de la cantidad de cabezas de la Hidra. Es por ello que el siguiente resultado de Kirby y Paris [3] pudiera parecer al principio bastante desconcertante.

Teorema 1 Contra cualquier Hidra inicial $H_{0}$, toda estrategia de Hércules es una estrategia ganadora.

¡Sorprendente! El teorema nos dice que en realidad Hércules no necesita emplear inteligencia alguna para vencer a la Hidra, por más monstruosa que inicialmente ésta sea.

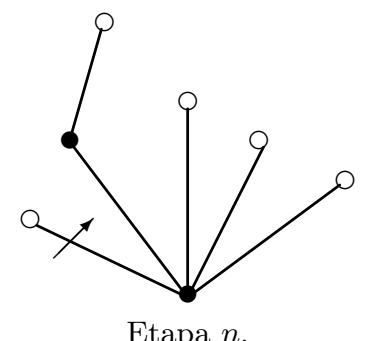

Etapa $n$.

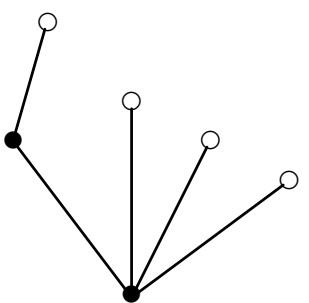

Etapa $n+1$.

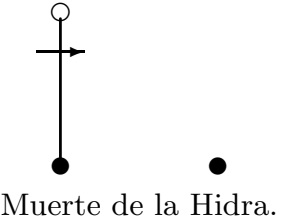

Figura 5: Al cortar una cabeza sin cuerpo la Hidra no reproduce nada. La muerte de la Hidra acontece cuando le cortan su última cabeza. 
Tan solo necesita tener paciencia y mantenerse vivo en el fragor de la batalla. Todavía más sorprendente es el siguiente resultado, debido también a Kirby y Paris [3].

Teorema 2 La afirmación "Contra cualquier Hidra inicial $H_{0}$, toda estrategia recursiva de Hércules es una estrategia ganadora" es una propiedad indemostrable en la Aritmética de Peano.

Es decir, el Teorema 1 - aún cuando es verdadero - no se puede demostrar empleando las técnicas tradicionales de la aritmética. Cuando hablamos de una estrategia recursiva nos referimos a una estrategia en la cual Hércules va escogiendo las cabezas de acuerdo a algún método o algoritmo preciso, método que, por ejemplo, no admite la escogencia al azar.

Demostraremos más adelante (Teorema 4) una generalización del Teorema 1. La demostración del Teorema 2 es sumamente compleja, enmarcándose dentro de las técnicas de la Teoría de Modelos, y requiere del empleo del concepto de "conjuntos finitos $\alpha$-largos", maquinaria desarrollada por Ketonen y Solovay [2]. Kirby y Paris demostraron que al menos existe una estrategia recursiva que - a pesar que es ganadora - no se puede demostrar este hecho dentro de la Aritmética de Peano. Simplificando, podemos afirmar que la imposibilidad de demostrar en Teorema 1 dentro de la Aritmética de Peano radica en el hecho que existen estrategias recursivas donde el número de etapas necesarias para darle muerte a la Hidra es inmenso, mayor que el crecimiento de las funciones primitivorecursivas.

Pero primeramente vamos a introducir un patrón de crecimiento similar, las interesantes sucesiones de Goodstein, estrechamente ligadas al crecimiento de las cabezas de las Hidras y utilizadas por Kirby \& Paris en las demostraciones de estos resultados.

\section{Sucesiones de Goodstein}

Empecemos la discusión con el siguiente ejemplo. El número 266 admite una única representación binaria:

$$
266=2^{8}+2^{3}+2^{1}
$$

En la representación anterior, los exponentes 8 y 3 también podemos representarlos en base 2, y también los exponentes de los exponentes, etc., para obtener la representación estricta en base 2 de 266 :

$$
266=2^{2^{2+1}}+2^{2+1}+2^{1} .
$$

Definimos la sucesión $\left(266_{k}\right)_{k \in \mathbf{N}}$ de la siguiente forma: el primer término es $266_{0}=$ $266=2^{2^{2+1}}+2^{2+1}+2^{1}$. Construimos el siguiente término intercambiando cada ocurrencia de 2 en la representación estricta de $266_{0}$ por un 3 y luego restando 1 , para obtener:

$$
\begin{aligned}
266_{1} & :=3^{3^{3+1}}+3^{3+1}+3^{1}-1 \\
& =3^{3^{3+1}}+3^{3+1}+2 \quad\left(\approx 0.44 \times 10^{39}\right) .
\end{aligned}
$$


De igual manera continuamos construyendo el siguiente término $266_{2}$ de nuestra sucesión, intercambiando cada ocurrencia de 3 en la representación estricta de 266 1 por un 4 y luego restando 1, para obtener:

$$
266_{2}:=4^{4^{4+1}}+4^{4+1}+1 \quad\left(\approx 0.32 \times 10^{617}\right) .
$$

Los siguientes dos términos $266_{3}$ y $266_{4}$ no requieren explicación:

$$
\begin{aligned}
266_{3} & :=5^{5^{5+1}}+5^{5+1}+0 \quad\left(\approx 0.25 \times 10^{10918}\right) \\
266_{4} & :=6^{6^{6+1}}+6^{6+1}-1 \\
& =6^{6^{6+1}}+6^{6} \cdot 5+6^{5} \cdot 5+6^{4} \cdot 5+6^{3} \cdot 5+6^{2} \cdot 5+6^{1} \cdot 5+5 \quad\left(\approx 0.35 \times 10^{217833}\right) .
\end{aligned}
$$

En el ejemplo hemos empezado con el número $m=266$ y la base $n=2$. Podemos generalizar el ejemplo empezando en un número cualquiera $m \in \mathbf{N}$ y una base arbitraria $n \in \mathbf{N}, n \geq 2$. Obtenemos la sucesión general de Goodstein $\left(m_{k}\right)_{k \in \mathbf{N}}$.

Definición 5 (Representación estricta en base $n$ ) Sean $m, n \in \mathbf{N}$, con $n \geq 2$. Denotamos por $\{m\}_{n}$ la representación estricta de $m$ en base $n$, esto es, la representación de $m$ en base $n$ en la cual los exponentes y los exponentes de los exponentes son todos representados en base $n$.

En la representación estricta $\{m\}_{n}$ el proceso de codificar los exponentes - y los exponentes de los exponentes - todos en base $n$ es finito, como el lector puede fácilmente observar.

Definición 6 (Sucesión general de Goodstein) Sean $m, n \in \mathbf{N}$, con $n \geq 2$. Definimos la sucesión general de Goodstein $\left(m_{k}\right)_{k \in \mathbf{N}}$ que empieza en la base $n$, mediante:

$$
\begin{aligned}
& m_{0}:=m \\
& m_{k}:=G_{n+k-1}\left(m_{k-1}\right), \quad \forall k \in \mathbf{N}^{*},
\end{aligned}
$$

donde $G_{n}(m)$ es el número obtenido al intercambiar dentro de $\{m\}_{n}$ cada ocurrencia de $n$ por $n+1$ y restar 1 .

Por ejemplo, para $n=2$ (empezando en la base 2), tendremos $m_{1}:=G_{2}\left(m_{0}\right), m_{2}:=$ $G_{3}\left(m_{1}\right), \ldots$ etc., que produce la sucesión de Goodstein estándar $\left(m_{k}\right)_{k \in \mathbf{N}}$, la cual hemos visto tiene un crecimiento inicial desorbitado. Sorprende el siguiente resultado, el cual establece entre otras cosas que cualquier sucesión de Goodstein converge a 0, a pesar del crecimiento inicial.

Teorema 3 Para cualquier base inicial $n \geq 2$ :

(a) (Goodstein, 1944): $(\forall m)\left[(\exists k)\left[m_{k}=0\right]\right]$

(b) (Ketonen y Solovay, 1981): $(\forall m)\left[(\exists k)\left[m_{k}=0\right]\right]$ es una propiedad indemostrable en la Aritmética de Peano. 
Demostración de (a): Básicamente la siguiente demostración es de Kirby y Paris (ver [3]). Con la sucesión general de Goodstein $m_{0}, m_{1}, m_{2}, \ldots$ asociamos una nueva sucesión estrictamente decreciente de números ordinales ${ }^{9} \alpha_{0}, \alpha_{1}, \alpha_{2}, \ldots$ tal que cada $\alpha_{k}<\epsilon_{0}=$ $\left.\omega^{\omega^{\cdots \omega}}\right\} \omega$ veces. Intuitivamente:

$$
\begin{aligned}
& 266_{0}=2^{2^{2+1}}+2^{2+1}+2^{1} \longleftrightarrow \alpha_{0}:=\omega^{\omega^{\omega+1}}+\omega^{\omega+1}+\omega \\
& 266_{1}=3^{3^{3+1}}+3^{3+1}+2 \longleftrightarrow \alpha_{1}:=\omega^{\omega^{\omega+1}}+\omega^{\omega+1}+2 \\
& 266_{2}=4^{4^{4+1}}+4^{4+1}+1 \longleftrightarrow \alpha_{2}:=\omega^{\omega^{\omega+1}}+\omega^{\omega+1}+1 \\
& 266_{3}=5^{5^{5+1}}+5^{5+1} \quad \longleftrightarrow \alpha_{3}:=\omega^{\omega^{\omega+1}}+\omega^{\omega+1}
\end{aligned}
$$

Luego, del principio de inducción transfinita por debajo de $\epsilon_{0}$, existe $k \in \mathbf{N}$ tal que $\alpha_{k}=0$. Ello implica que $m_{k}=0$. Solamente resta formalizar algunas cosas:

1. Primitivo-recursividad de la sucesión de Goodstein $\{m\}_{n}$ : Sea

$$
m=\sum_{i=0}^{s} n^{i} a_{i}=n^{s} a_{s}+n^{s-1} a_{s-1}+\cdots+n a_{1}+a_{0}
$$

la representación de $m$ en base $n$. Definimos la función $g^{m, n}: \mathbf{N} \mapsto \mathbf{N}$ mediante la recursión

$$
g^{m, n}(x):= \begin{cases}\sum_{i=0}^{s} x^{g^{i, n}(x)} a_{i} & , \text { si } m>0 \\ 0 & \text {, si } m=0\end{cases}
$$

Entonces $g^{m, n}$ es primitivo-recursiva y además $\{m\}_{n}=g^{m, n}(n)$. Definimos la sucesión de Goodstein $\left(m_{k}\right)$ que empieza en la base $n$, mediante:

$$
\begin{aligned}
& m_{0}:=m, \\
& m_{1}:=G_{n}\left(m_{0}\right)=g^{m_{0}, n}(n+1)-1,
\end{aligned}
$$

y en general,

$$
m_{k+1}:=G_{n+k}\left(m_{k}\right)=g^{m_{k}, n+k}(n+k+1)-1, \quad \forall k \in \mathbf{N} .
$$

2. Manera exacta de asociar ordinales a cada $m_{k}$ : Considérese el operador de ordinales $\langle\alpha\rangle(n)$, definido para $\alpha \in \mathcal{O}$ rd, $n \in \mathbf{N}, \alpha<\epsilon_{0}$, inductivamente mediante:

$$
\begin{aligned}
\langle 0\rangle(n) & :=0, \\
\langle\beta+1\rangle(n) & :=\beta, \quad \mathrm{y} \operatorname{para} \delta>0 \\
\left\langle\omega^{\delta} \cdot(\beta+1)\right\rangle(n) & :=\omega^{\delta} \cdot \beta+\omega^{\langle\delta\rangle(n)} \cdot n+\left\langle\omega^{\langle\delta\rangle(n)}\right\rangle(n) .
\end{aligned}
$$

\footnotetext{
${ }^{9}$ Los números ordinales $\mathcal{O}$ rd son una extensión dentro del infinito de las propiedades de orden de los números naturales $0,1, \ldots$ La clase $\mathcal{O}$ rd no es propiamente un conjunto y contiene números tales como $\omega, \omega^{2}+\omega+1$, etc, donde $\omega=(\mathbf{N}, \leq)$ denota el primer infinito. Con la aritmética de los números ordinales tendremos cosas tales como $\omega+1 \neq 1+\omega$, o como $2^{\omega}=\omega$, o $1+\omega=\omega$, etc. Al lector sin experiencia en este tema de los números ordinales se le recomienda una primera lectura en el libro de Monk [8].
} 
Podemos agregar formalmente $\omega$ al dominio de las funciones $g^{m, n}$, para obtener:

$$
\begin{aligned}
m_{0} & \longleftrightarrow \alpha_{0}:=g^{m_{0}, n}(\omega), \\
m_{1} & \longleftrightarrow \alpha_{1}:=\left\langle\alpha_{0}\right\rangle(n), \quad \text { y en general, } \\
m_{k+1} & \longleftrightarrow \alpha_{k+1}:=\left\langle\alpha_{k}\right\rangle(n+k), \quad \forall k \in \mathbf{N} .
\end{aligned}
$$

De la definición es claro que $\left(\alpha_{k}\right)$ es una sucesión estrictamente decreciente y además $\alpha_{k}<\epsilon_{0}$, para todo $k \in \mathbf{N}$. Luego, el principio de inducción transfinita es aplicable a $\left(\alpha_{k}\right)$, concluyéndose la demostración de (a).

Idea de la demostración de (b): El Teorema de Goodstein no puede ser demostrado en la Aritmética de Peano fundamentalmente debido al tiempo desproporcionado que le toma a la sucesión $\left(m_{k}\right)$ alcanzar 0 . En efecto, sea $g_{n}: \mathbf{N} \mapsto \mathbf{N}$ la función definida por $g_{n}(m):=\mu k\left[m_{k}=0\right]$, esto es, el menor índice $k$ para el cual la sucesión de Goodstein $\left(m_{k}\right)$ empezando en la base $n$ alcanza a 0 . Claramente las funciones $g_{n}$ son recursivas y son también totales, para todo $n \geq 2$, debido a la veracidad del Teorema de Goodstein.

Ketonen y Solovay demostraron, a través de métodos indirectos relacionados con la teoría de indicadores, que bajo la suposición que el Teorema de Goodstein fuese demostrable dentro de la Aritmética de Peano, entonces para cualquier función recursiva y total $f$ existe $n \in \mathbf{N}$ tal que $f(x)<g_{n}(x)$, para $x$ suficientemente grande. Esto nos lleva a un absurdo, pues con lo anterior llegaríamos a construir una función recursiva y total $g$ que crezca más rápido que cualquier función recursiva y total.

Incidentalmente se menciona que aunque las funciones $g_{n}$ son recursivas y totales, sin embargo no son primitivo-recursivas y en consecuencia sus crecimientos son desmesurados. Por ejemplo, la sucesión estándar (empezando en la base 2) $\left(4_{k}\right)$ alcanza a 0 hasta el astronómico índice de $k=g_{2}(4)=3 \times 2^{402653211}-3$, un número del orden de $10^{121210700}$. Los primeros términos de esta sucesión $\left(4_{k}\right)$ se exhiben en la Figura 6 .

\section{Números ordinales asociados a las Hidras}

Definición 7 Sea $H$ una Hidra. Podemos asociar a $H$ con un número ordinal $\alpha=\alpha(H)$ de acuerdo al siguiente procedimiento recursivo: a cada nodo $s \in H$ le asociamos un número ordinal:

(a) Si s es una cabeza, le asociamos el número ordinal 0.

(b) Si s no es una cabeza, entonces $s$ tiene digamos $k$ nodos sucesores inmediatos. Sean $\beta_{1} \geq \beta_{2} \geq \cdots \geq \beta_{k}$ los números ordinales asociados a estos sucesores inmediatos. Entonces asociamos a s el número ordinal $\omega^{\beta_{1}}+\omega^{\beta_{2}}+\cdots+\omega^{\beta_{k}}$.

Finalmente, $\alpha(H)$ será el ordinal asociado al nodo raíz $(H)$.

En la Figura 7 se ilustra este procedimiento. Varios comentarios deben hacerse. Primeramente se observa que para cualquier Hidra $H$ tendremos $\alpha(H)<\epsilon_{0}$, en virtud que $H$ es un árbol finito. Recíprocamente, si $\alpha<\epsilon_{0}$ es un ordinal cualquiera, entonces podemos 


\begin{tabular}{rlll} 
Sucesión de Goodstein & & Ordinal $\alpha_{k}$ asociado \\
\hline $4_{0}=2^{2}$ & $\longleftrightarrow$ & $\omega^{\omega}$ \\
$4_{1}=3^{3}-1=3^{2} \cdot 2+3^{1} \cdot 2+2$ & $\longleftrightarrow$ & $\omega^{2} \cdot 2+\omega \cdot 2+2$ \\
$4_{2}=4^{2} \cdot 2+4^{1} \cdot 2+1$ & $\longleftrightarrow$ & $\omega^{2} \cdot 2+\omega \cdot 2+1$ \\
$4_{3}=5^{2} \cdot 2+5^{1} \cdot 2$ & $\longleftrightarrow$ & $\omega^{2} \cdot 2+\omega \cdot 2$ \\
$4_{4}=6^{2} \cdot 2+6^{1} \cdot 2-1=6^{2} \cdot 2+6^{1}+5$ & $\longleftrightarrow$ & $\omega^{2} \cdot 2+\omega+5$ \\
$4_{5}=7^{2} \cdot 2+7^{1}+4$ & $\longleftrightarrow$ & $\omega^{2} \cdot 2+\omega+4$ \\
$\vdots$ & $\vdots$ & & $\vdots$ \\
$4_{9}=11^{2} \cdot 2+11^{1}+0$ & $\longleftrightarrow$ & $\omega^{2} \cdot 2+\omega$ \\
$4_{10}=1^{2} \cdot 2+12^{1}-1$ & $\longleftrightarrow$ & $\omega^{2} \cdot 2+11$ \\
$4_{11}=1^{2} \cdot 2+10$ & & $\omega^{2} \cdot 2+10$ \\
$\vdots$ & $\vdots$ & & $\vdots$ \\
$4_{21}=2^{2} \cdot 23^{2} \cdot 2+0$ & & $\omega^{2} \cdot 2$ \\
$4_{22}=24^{2} \cdot 2-1=24^{2}+24 \cdot 23+23$ & $\longleftrightarrow$ & $\omega^{2}+\omega \cdot 23+23$ \\
$4_{23}=2^{2}+25 \cdot 23+25$ & & $\vdots$ \\
$\vdots$ & $\vdots$ & &
\end{tabular}

Figura 6: Primeros términos de la sucesión estándar de Goodstein $\left(4_{k}\right)$.

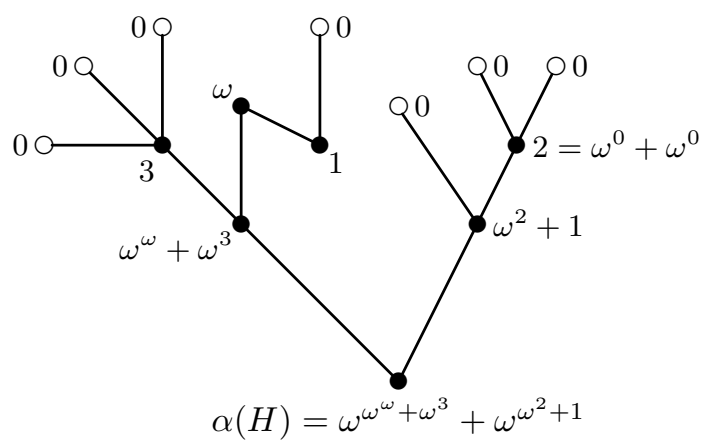

Figura 7: Procedimiento para asociar un número ordinal a la Hidra $H$. 
asociar $\alpha$ con una Hidra $H=H(\alpha)$. En efecto, debido a que $\alpha<\epsilon_{0}$, entonces vale la forma normal de Cantor, de manera que $\alpha$ se expresa de manera única como

$$
\alpha=\omega^{\alpha_{1}}+\omega^{\alpha_{2}}+\cdots+\omega^{\alpha_{r}}
$$

con $\alpha>\alpha_{1} \geq \alpha_{2} \geq \cdots \geq \alpha_{r}$ y además al expresar cada ordinal $\alpha_{1}, \alpha_{2}, \ldots, \alpha_{r}$ mediante la misma forma normal de Cantor, el proceso se estabiliza luego de un número finito de etapas. Por ejemplo, los números ordinales $\alpha$ y $\beta$ siguientes

$$
\alpha=\omega^{\omega^{\omega+1}+\omega^{3}+1}+\omega^{\omega^{2}+1}+1 \quad, \quad \beta=\omega^{\omega^{\omega}}+\omega^{\omega^{3} \cdot 2+1}+\omega \cdot 3+1
$$

definen las Hidras $H(\alpha)$ y $H(\beta)$ ilustradas en la Figura 8. Está claro que para cualquier número ordinal $\alpha<\epsilon_{0}$ tendremos las identidades $\alpha(H(\alpha))=\alpha$ y $H(\alpha(H))=H$, si identificamos como "iguales" (o "equivalentes") las Hidras que sólo difieren en el orden de sus nodos.
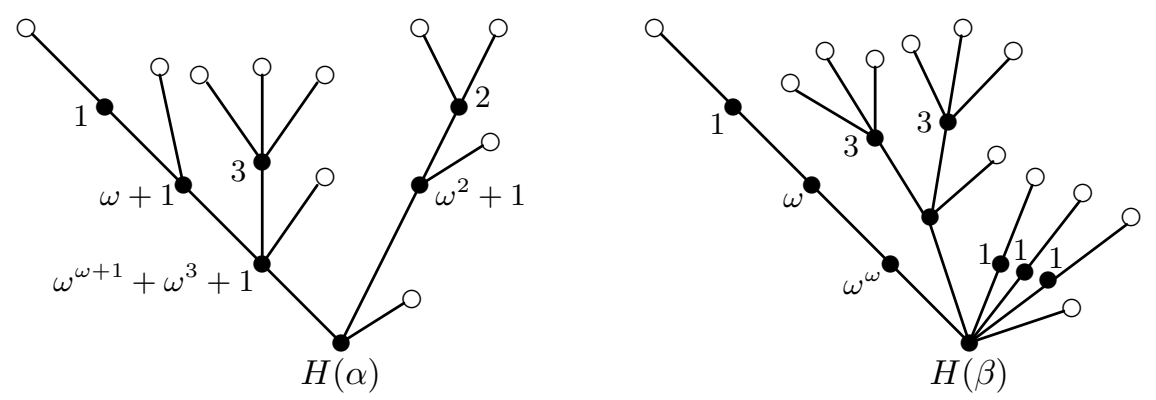

Figura 8: Construcción de las Hidras correspondientes a los números ordinales $\alpha$ y $\beta$ dados.

\section{$5 \quad$ Estrategias más lenta y más corta}

Hemos visto que en la batalla entre Hércules y la Hidra, cualquier estrategia que Hércules emplee es ganadora. ¿Cuál es la estrategia $\tau_{\max }$ más lenta? ¿Y cuál es la estrategia $\tau_{\min }$ más corta? Para una Hidra $H$, estas estrategias "optimales" se definen como sigue:

$\tau_{\max }$ : Consiste en escoger, en cada etapa, la cabeza que se encuentre "más cercana" a la raíz, esto es, ascender desde la raíz de $H$ por el subárbol asociado con número ordinal más pequeño. En caso de empates, se escoge cualquier cabeza entre las que produzcan empate.

$\tau_{\min }$ : Consiste en escoger, en cada etapa, la cabeza que se encuentre "más alejada" de la raíz, esto es, ascender desde la raíz de $H$ por el subárbol asociado con número ordinal más grande. En caso de empates, se escoge cualquier cabeza entre las que produzcan empate. 

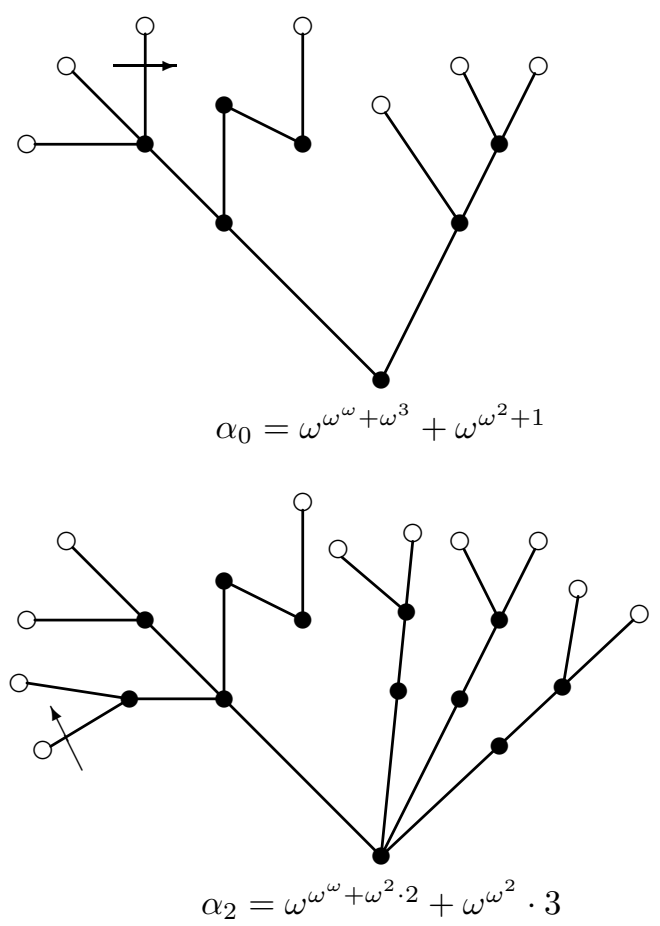
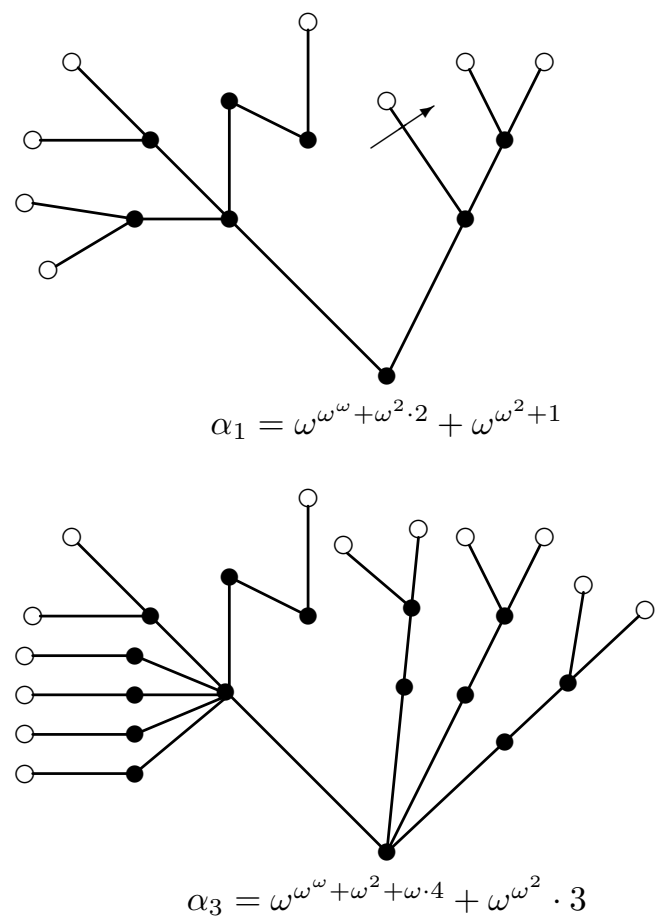

Figura 9: Sucesión $\left(\alpha_{n}\right)$ de ordinales asociados a las Hidras en la batalla de la Figura 4.

Pues bien, Loebl demostró en [4] que mientras la estrategia $\tau_{\text {min }}$ produce batallas con duración (número de etapas hasta que la Hidra es aniquilada) primitivo-recursivo, esto es, batallas "cortas", por el contrario la estrategia $\tau_{\max }$ produce batallas con una duración superior al crecimiento de las funciones primitivo-recursivas, esto es, batallas "largas". Por otra parte, Miserscque demostró en [7] que para cualquier Hidra $H$, la estrategia $\tau_{\text {max }}$ produce la batalla más larga entre Hércules y la Hidra $H$. Precisamente la finitud de la batalla al emplear esta estrategia $\tau_{\max }$ es indemostrable dentro de la Aritmética de Peano.

Matousek y Loebl estudiaron en [6] el siguiente juego de estrategias mixtas: supongamos que la Hidra tiene un ayudante oculto, que procura alargar la batalla lo más posible. Cada vez que Hércules realiza una "movida" cortando una cabeza (buscando matar al monstruo lo más rápido posible), entonces le siguen $k$ diferentes "movidas" del ayudante oculto de la Hidra, del mismo tipo que las de Hércules aunque buscando mantener a la Hidra viva el mayor tiempo posible. Demostraron que cuando $k=1$ Hércules puede mantener la batalla "corta" (esto es, con una duración primitivo-recursiva como función del número de nodos de la Hidra inicial), mientras que si $k \geq 2$ el ayudante oculto de la Hidra tiene una estrategia para hacer la batalla "larga" (duración superior al crecimiento de las funciones primitivo-recursivas, o finitud no demostrable dentro de la Aritmética de Peano). 


\section{Modelo alternativo en la reproducción de las cabezas}

En el modelo clásico introducido por Kirby y Paris, a la Hidra le van brotando las cabezas siguiendo una progresión aritmética: en la primera etapa le brota 1 copia del cuerpo de la cabeza cortada; en la segunda etapa le brotan 2 copias del cuerpo; en la tercera etapa le brotan 3 copias del cuerpo; etc.

Una de las primeras críticas que le pueden surgir al lector es. . ¿¿Porqué tan organizada esta ley de reproducción de las cabezas, siguiendo una progresión aritmética? ¿Porqué mejor no seguir una progresión geométrica o un crecimiento de cualquier otro tipo en este asunto de la reproducción de las cabezas?

La finitud de la batalla entre Hércules y la Hidra no se ve afectada cuando se cambia el modelo clásico por uno alternativo que permita una reproducción de una cantidad arbitraria de las cabezas. Este es un resultado propio del autor y se expone a continuación.

Teorema 4 Supóngase que la ley de reproducción de las cabezas en el modelo de Kirby y Paris es modificada de acuerdo al siguiente patrón: en la etapa n-ésima luego del corte de una cabeza, le brotan a la Hidra $f(n)$ réplicas del cuerpo de la cabeza cortada, donde $f: \mathbf{N} \mapsto \mathbf{N}$ es una función total. Entonces, para cualquier Hidra inicial $H$, toda estrategia de Hércules es ganadora.

Demostración: La batalla consiste en una sucesión de Hidras $\left(H_{n}\right)_{n \in \mathbf{N}}$, a las cuales asociamos los números ordinales correspondientes $\left(\alpha_{n}\right)_{n \in \mathbf{N}}$, donde $\alpha_{n}:=\alpha\left(H_{n}\right)$, siguiendo el procedimiento descrito para asignar números ordinales a las Hidras.

Sea $\tau$ una estrategia de Hércules. Definimos el operador de ordinales $[\alpha]_{\tau}(n)$ que calcula el número ordinal $\alpha_{n}$ en términos del anterior ordinal $\alpha_{n-1}$ y el natural $n$, esto es, $\alpha_{n}:=\left[\alpha_{n-1}\right]_{\tau}(n)$, empleando la estrategia $\tau$. Vamos a demostrar que la sucesión $\left(\alpha_{n}\right)$ es estrictamente decreciente, pues entonces aplicamos el principio de inducción transfinita por debajo de $\epsilon_{0}$ para deducir la existencia de un índice $k \in \mathbf{N}$ para el cual $\alpha_{k}=0$, significando que la Hidra $H_{k}$ correspondiente ha muerto. Para demostrar la decrecencia de $\left(\alpha_{n}\right)$ basta probar que para cualquier $\alpha \in \mathcal{O}$ rd, con $0<\alpha<\epsilon_{0}$ (esto es, frente a cualquier Hidra) e independientemente de las cabezas elegidas por la estrategia $\tau$, tendremos

$$
[\alpha]_{\tau}(n)<\alpha, \quad \forall n \in \mathbf{N}
$$

Demostraremos esto empleando inducción transfinita sobre $\alpha$ por debajo de $\epsilon_{0}$, examinando las cinco formas diferentes que $\alpha$ podría tener, de acuerdo a la teoría de los números ordinales y el nuevo esquema de reproducción propuesto. Primeramente debe observarse que para algunos ordinales $\alpha<\epsilon_{0}$ relativamente simples, el valor de $[\alpha]_{\tau}(n)$ es independiente de la estrategia $\tau$ elegida. En efecto, de la definición de la reproducción de las cabezas de las Hidras, tendremos los siguientes casos particulares:

$$
\begin{aligned}
{[0]_{\tau}(n) } & =0, \\
{[a+1]_{\tau}(n) } & =a, \\
{\left[\omega^{\gamma+1} \cdot(a+1)\right]_{\tau}(n) } & =\omega^{\gamma+1} \cdot a+\omega^{\gamma} \cdot f(n),
\end{aligned}
$$


para todo $\gamma<\epsilon_{0}, a \in \mathbf{N}$ y $n \in \mathbf{N}$. Está claro que los casos iniciales no triviales (3) y (4) cumplen con la propiedad (1). A continuación supongamos (como hipótesis de inducción) que la propiedad (1) se satisface para todos los ordinales $\beta$ menores que $\alpha$. Quedan por analizar dos posibles formas que puede tener $\alpha$. La primera de ellas es cuando $\alpha$ tiene la forma $\omega^{\delta} \cdot(a+1)$, donde $\delta$ es un ordinal límite. En tal caso, tendremos:

$$
\left[\omega^{\delta} \cdot(a+1)\right]_{\tau}(n)=\omega^{\delta} \cdot a+\omega^{[\delta]_{\tau}(n)},
$$

y al ser $\delta<\alpha$, entonces la hipótesis de inducción es aplicable, demostrándose la propiedad (1) para cualquier estrategia $\tau$ que se emplee. La última posibilidad en cuanto a la forma del ordinal $\alpha$ surge cuando éste tiene la forma

$$
\alpha=\omega^{\lambda_{1}} \cdot\left(a_{1}+1\right)+\cdots+\omega^{\lambda_{i}} \cdot\left(a_{i}+1\right)+\cdots+\omega^{\lambda_{r}} \cdot\left(a_{r}+1\right),
$$

con $\alpha>\lambda_{1}>\cdots>\lambda_{r}$ y $a_{i} \in \mathbf{N}, 1 \leq i \leq n$. La estrategia $\tau$ se manifiesta en la escogencia de alguno de los subárboles asociados a los anteriores sumandos. Supongamos que $\tau$ consiste en escoger el subárbol que comienza con el $i$-ésimo sumando, $\omega^{\lambda_{i}} \cdot\left(a_{i}+1\right)$. Luego, tendremos:

$$
[\alpha]_{\tau}(n)=\omega^{\lambda_{1}} \cdot\left(a_{1}+1\right)+\cdots+\omega^{\left[\lambda_{i}\right]_{\tau}(n)} \cdot\left(a_{i}+1\right)+\cdots+\omega^{\lambda_{r}} \cdot\left(a_{r}+1\right) .
$$

Pero como $\lambda_{i}<\alpha$, aplicamos la hipótesis de inducción para obtener $\left[\lambda_{i}\right]_{\tau}(n)<\lambda_{i}$, de donde se deduce la propiedad (1).

La técnica de demostración empleada es similar a la utilizada por Misercque en [7] y sigue las ideas centrales de Kirby y Paris [3]. Puede demostrarse que en el modelo generalizado aquí descrito, el número de movidas que requerirá Hércules para matar a la Hidra es recursiva en $f$, como función del número de nodos de la Hidra inicial.

Otro interesante problema relacionado con el modelo estándar de Kirby y Paris sobre Hércules y la Hidra es examinar si existe una prueba dentro de la Aritmética de Peano de la finitud de la estrategia recursiva $\tau_{\min }$. Pues bien, jeste es aún un problema abierto!

En efecto, el Teorema 2 establece tan solo que existen estrategias recursivas $\tau$ que no admiten prueba de finitud dentro de la Aritmética de Peano. Sin embargo, aún en el modelo clásico de Kirby y Paris el problema de probar la existencia de estrategias recursivas cuya finitud sea demostrable dentro de la aritmética usual podría llegar a ser tan difícil como la famosa conjetura $\mathcal{P} \neq \mathcal{N} \mathcal{P}$, aún no resuelta y fundamental en las Ciencias de la Computación.

Algunos casos particulares de este problema ya fueron resueltos: en efecto, Luccio y Pagli en [5] estudiaron el caso particular del esquema alternativo de reproducción de cabezas propuesto en el Teorema 4, cuando $f(n)=2$ para todo $n \in \mathbf{N}$ (esto es, cuando la Hidra reproduce en cada etapa dos copias del cuerpo de la cabeza cortada). Hallaron una prueba combinatoria de la finitud de $\tau_{\text {min }}$ dentro de la aritmética usual, empleando la noción de potencial de los nodos la Hidra. Su demostración puede extenderse al caso de cualquier función acotada, recursiva y total $f$. 


\section{$7 \quad$ La muerte del Internet ${ }^{10}$}

Consideremos ahora un monstruo diferente: la red Internet, un gigantesco e intrincado conjunto de portales (sites), que promete ser el futuro de la globalización comercial. En una primera aproximación podemos ver la estructura de este monstruo representada como un árbol dinámico, donde un usuario encuentra más y más páginas especializadas moviéndose de la raíz hacia las hojas, y tales páginas son actualizadas continuamente. Para discutir este fenómeno podríamos visitar una compañía de reciente creación en la dirección

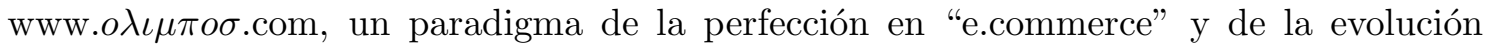
que ha tenido la Internet (Figura 10). Tres viejos departamentos de ventas han sido establecidos desde hace algún tiempo, bajo la supervisión de Afrodita ${ }^{11}$ (Departamento de Belleza), Atenea (Armería) y Hermes (Ferretería), y un nuevo departamento ha sido abierto recientemente bajo la supervisión de Dioniso ${ }^{12}$ (Vinos y Licores), accesible por el momento justamente como una página del árbol de $o \lambda \iota \mu \pi \circ \sigma$.

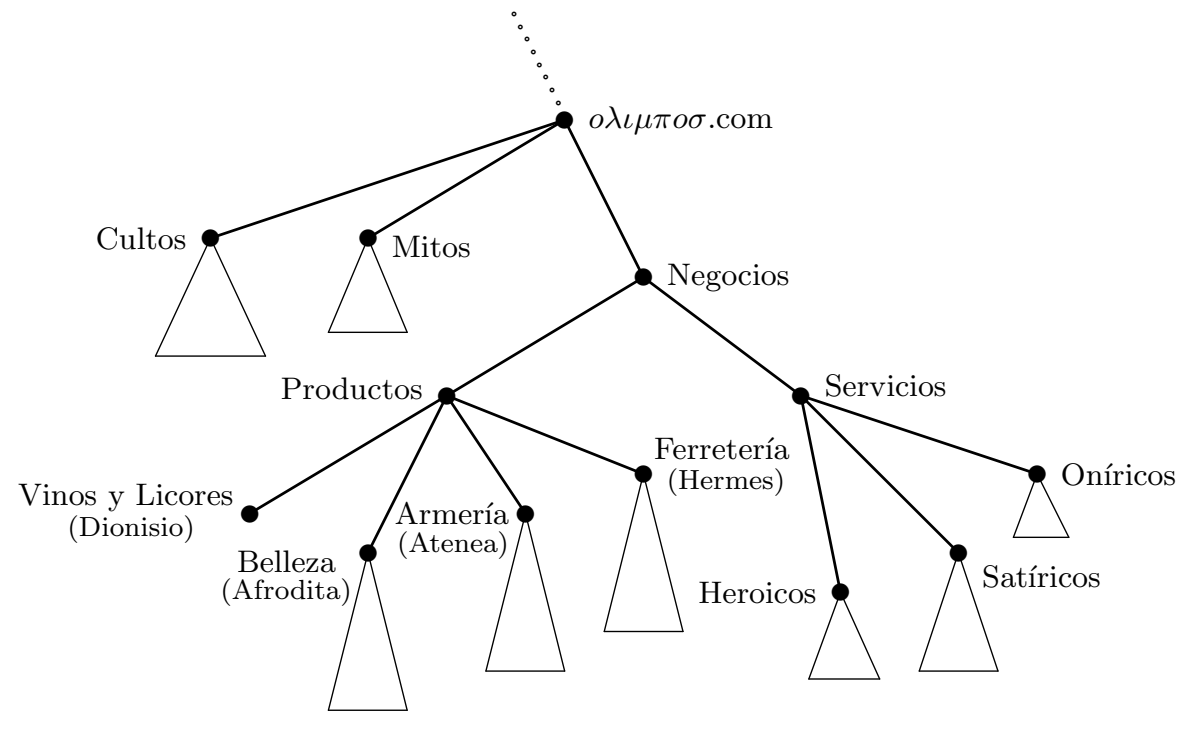

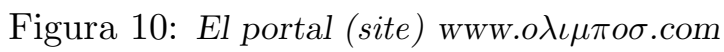

Pero hoy día las bebidas espirituosas adquieren mayor demanda y el número de accesos a la página de Dioniso se ha incrementado tan rápidamente que la compañía ha decidido actualizar su portal (site). La página de Vinos y Licores será movida un nivel para arriba en el árbol para que sea más accesible, pasando a ser un hijo de Negocios y hermana de Productos. Alguna estructura también se le adicionará a la descripción del departamento, del mismo tipo de la que ha sido experimentada exitosamente para Productos. Esto es, un subárbol como el de Productos será creado para Vinos y Licores.

\footnotetext{
${ }^{10}$ Alegoría de Fabriccio Luccio y Linda Pagli (en [5]) de la Universidad de Pisa, Italia, producto de las disquisiciones en torno a las ideas de una conferencia que ofreció el autor sobre tema de Hércules y la Hidra, en La Habana, Cuba, en enero del año 1999.

${ }^{11}$ Afrodita es la diosa de la Belleza y del Amor, identificada con la Venus de los romanos.

${ }^{12}$ Dioniso es el dios griego del Vino, hijo de Zeus y de Semele, identificado con el Baco de los romanos.
} 
Hasta aquí todo bien. Pero el árbol experimentará una transformación del tipo Hidra, siguiendo el patrón de crecimiento precisamente considerado en el Teorema 4 cuando $f(n)=1$. Entonces tendremos la conclusión inevitable: jel Internet va a desaparecer!

Epílogo. En efecto, el Internet eventualmente desaparecerá. En vista que esta conclusión podría generar pánico en Wall Street, nos sentimos obligados a agregar algunas pocas palabras. Primero, la aniquilación tomará billones de etapas, de manera que no hay peligro en el corto o mediano plazo. Segundo, cuando una hoja asociada directamente con la raíz del árbol es cortada, nada crece del árbol, aunque sin embargo podemos estar seguro que un árbol paralelo florecerá en alguna otra parte del mundo. Una miríada de nuevas Internets aparecerá, luego desaparecerá, etc. ¡Esté preparado!

\section{Referencias}

[1] Goodstein, R. L. (1944) "On the restricted ordinal theorem", Journal of Symbolic Logic 9: 33-41.

[2] Ketonen, J.; Solovay, R. (1981) "Rapidly growing Ramsey functions", Annals of Mathematics 113: 267-314.

[3] Kirby, L.; Paris, J. (1982) "Accessible independence results for Peano arithmetic", Bulletin of the London Mathematical Society 14: 285-293.

[4] Loebl, M. (1988) "Hercules and Hydra", Commentationes Mathematicae Univesitatis Carolinae 29(1), 85-95.

[5] Luccio, F.; Pagli, L. (2000) "Death of a monster", Pre-print, Departamento de Informática de la Universidad de Pisa, Italia.

[6] Matousek, J.; Loebl, M. (1991) "Hercules versus hidden Hydra helper", Commentationes Mathematicae Univesitatis Carolinae 32(4): 731-741.

[7] Misercque, D. (1990) "Le plus long combat d'Hercule", Bulletin de la Société Mathématique de Belgique 42, série B: 319-331.

[8] Monk, D.J. (1969) Introduction to Set Theory. McGraw-Hill Book Company, New York.

[9] Rogers, H. (1967) Theory of Recursive Functions and Effective Computability. McGraw-Hill Book Company, New York. 